\title{
Sunlet Decomposition of Certain Equipartite Graphs
}

\author{
Abolape D. Akwu' ${ }^{1}$ and Deborah O. A. Ajayi ${ }^{2}$ \\ ${ }^{1}$ Department of Mathematics, University of Agriculture, Makurdi 970001, Nigeria \\ ${ }^{2}$ Department of Mathematics, University of Ibadan, Ibadan 200001, Nigeria
}

Correspondence should be addressed to Deborah O. A. Ajayi; adelaideajayi@yahoo.com

Received 28 September 2012; Accepted 5 February 2013

Academic Editor: Chris A. Rodger

Copyright (C) 2013 A. D. Akwu and D. O. A. Ajayi. This is an open access article distributed under the Creative Commons Attribution License, which permits unrestricted use, distribution, and reproduction in any medium, provided the original work is properly cited.

Let $L_{2 n}$ stand for the sunlet graph which is a graph that consists of a cycle and an edge terminating in a vertex of degree one attached to each vertex of cycle $C_{n}$. The necessary condition for the equipartite graph $K_{n}+I * \bar{K}_{m}$ to be decomposed into $L_{2 n}$ for $n \geq 2$ is that the order of $L_{2 n}$ must divide $n^{2} m^{2} / 2$, the order of $K_{n}+I * \bar{K}_{m}$. In this work, we show that this condition is sufficient for the decomposition. The proofs are constructive using graph theory techniques.

\section{Introduction}

Let $C_{r}, K_{n}, \bar{K}_{m}$ denote cycle of length $r$, complete graph on $n$ vertices, and complement of complete graph on $m$ vertices. For $n$ even, $K_{n}+I$ denotes the multigraph obtained by adding the edges of a 1 -factor to $K_{n}$, thus duplicating $n / 2$ edges. The total number of edges in $K_{n}+I$ is $n^{2} / 2$. The lexicographic product, $G * H$, of graphs $G$ and $H$, is the graph obtained by replacing every vertex of $G$ by a copy of $H$ and every edge of $G$ by the complete bipartite graph $K_{|H|,|H|}$.

For a graph $H$, an $H$-decomposition of a graph $G, H \mid G$, is a set of subgraphs of $G$, each isomorphic to $H$, whose edge set partitions the edge set of $G$. Note that for any graph $G$ and $H$ and any positive integer $m$, if $H \mid G$ then $\left(H * \bar{K}_{m}\right) \mid\left(G * \bar{K}_{m}\right)$.

Let $G$ be a graph of order $n$ and $H$ any graph. The corona (crown) of $G$ with $H$, denoted by $G \odot H$, is the graph obtained by taking one copy of $G$ and $n$ copies of $H$ and joining the $i$ th vertex of $G$ with an edge to every vertex in the $i$ th copy of $H$. A special corona graph is $C_{n} \odot K_{1}$, that is, a cycle with pendant points which has $2 n$ vertices. This is called sunlet graph and denoted by $L_{q}, q=2 n$.

Obvious necessary condition for the existence of a $k$-cycle decomposition of a simple connected graph $G$ is that $G$ has at least $k$ vertices (or trivially, just one vertex), the degree of every vertex in $G$ is even, and the total number of edges in $G$ is a multiple of the cycle length $k$. These conditions have been shown to be sufficient in the case that $G$ is the complete graph $K_{n}$, the complete graph minus a 1 -factor $K_{n}-I[1,2]$, and the complete graph plus a 1 -factor $K_{n}+I$ [3].

The study of cycle decomposition of $K_{n} * \bar{K}_{m}$ was initiated by Hoffman et al. [4]. The necessary and sufficient conditions for the existence of a $C_{p}$-decomposition of $K_{n} * \bar{K}_{m}$, where $p \geq 5$ ( $p$ is prime) that (i) $m(n-1)$ is even and (ii) $p$ divides $n(n-1) m^{2}$, were obtained by Manikandan and Paulraja $[5,6]$. Similarly, when $p \geq 3$ is a prime, the necessary and sufficient conditions for the existence of a $C_{2 p}$-decomposition of $K_{n} *$ $\bar{K}_{m}$ were given by Smith [7]. For a prime number $p \geq 3$, Smith [8] showed that $C_{3 p}$-decomposition of $K_{n} * \bar{K}_{m}$ exists if the obvious necessary conditions are satisfied. In [9], Anitha and Lekshmi proved that the complete graph $K_{n}$ and the complete bipartite graph $K_{n, n}$ for $n$ even have decompositions into sunlet graph $L_{n}$. Similarly, in [10], it was shown that the complete equipartite graph $K_{n} * \bar{K}_{m}$ has a decomposition into sunlet graph of length $2 p$, for a prime $p$.

We extend these results by considering the decomposition of $K_{n}+I * \bar{K}_{m}$ into sunlet graphs and prove the following result.

Let $m \geq 2, n>2$, and $q \geq 6$ be even integers. The graph $K_{n}+I * \bar{K}_{m}$ can be decomposed into sunlet graph of length $q$ if and only if $q$ divides $n^{2} m^{2} / 2$, the number of edges in $K_{n}+$ $I * \bar{K}_{m}$ 


\section{Proof of the Result}

To prove the result, we need the following.

Lemma 1 (see $[10])$. For $r \geq 3, L_{2 r}$ decomposes $C_{r} * \bar{K}_{2}$.

Lemma 2. For any integer $r>2$ and a positive even integer $m$, the graph $C_{r} * \bar{K}_{m}$ has a decomposition into sunlet graph $L_{q}$, for $q=r m$.

Proof

Case 1 ( $r$ is even). First observe that $C_{r} * \bar{K}_{2}$ can be decomposed into 2 sunlet graphs with $2 r$ vertices. Now, set $m=2 t$ and decompose $C_{r} * \bar{K}_{t}$ into cycles $C_{r t}$. To decompose $C_{r} * \bar{K}_{t}$ into $t$-cycles $C_{r t}$, denote vertices in $i$ th part of $C_{r} * \bar{K}_{t}$ by $x_{i, j}$ for $j=1, \ldots, t, i=1,2, \ldots, r$ and create $t$ base cycles $x_{1, j} x_{2, j} x_{3, j} \cdots x_{r-1, j} x_{r, j}$. Next, combine these base cycles into one cycle $C_{r t}$ by replacing each edge $x_{1, j} x_{2, j}$ with $x_{1, j} x_{2, j+1}$. To create the remaining cycles $C_{r t}$, we apply mappings $\phi_{s}$ for $s=0,1, \ldots, t-1$ defined on the vertices as follows.

Subcase 1.1 ( $i$ odd). Consider

$$
\phi_{s}\left(x_{i, j}\right)=x_{i, j}
$$

This is the desired decomposition into cycles $C_{r t}$.

Subcase 1.2 ( $i$ even). Consider

$$
\phi_{s}\left(x_{i, j}\right)=x_{i, j+s} .
$$

This is the desired decomposition into cycles $C_{r t}$.

Now take each cycle $C_{r t}$, and make it back into $C_{r t} * \bar{K}_{2}$. Each $C_{r t} * \bar{K}_{2}$ decomposes into 2 sunlet graphs $L_{2 r t}$ (by Lemma 1), and we have $C_{r} * \bar{K}_{m}$ decomposing into sunlet graphs with length $r m$ for $r$ even. Note that

$$
C_{r} * \bar{K}_{2 t}=\left(C_{r} * \bar{K}_{t}\right) * \bar{K}_{2} \text {. }
$$

Case 2 ( $r$ is odd)

Subcase $2.1(m \equiv 2(\bmod 4))$. Set $m=2 t$. First create $t$ cycles $C_{(r-1) t}$ in $C_{r-1} * \bar{K}_{t}$ as in Case 1 . Then, take complete tripartite graph $K_{t, t, t}$ with partite sets $X_{i}=\left\{x_{i, j}\right\}$ for $i=$ $1, r-1, r$ and $j=1, \ldots, t$ and decompose it into triangles using well-known construction via Latin square, that is, construct $t \times t$ Latin square and consider each element in the form $(a, b, c)$ where $a$ denotes the row, $b$ denotes the column, and $c$ denotes the entry with $1 \leq a, b, c \leq t$. Each cycle is of the form $x_{(1, a)}, x_{(r-1, b)}, x_{(r, c)}$. Then, for every triangle $x_{1, a} x_{r-1, b} x_{r, c}$, replace the edge $x_{1, a} x_{r-1, b}$ in each $C_{(r-1) t}$, by the edges $x_{r-1, b} x_{r, c}$ and $x_{r, c} x_{1, a}$ to obtain cycles $C_{r t}$. Therefore, $C_{r t} \mid C_{r} * \bar{K}_{t}$. Now take each cycle $C_{r t}$, make it into $C_{r t} * \bar{K}_{2}$, and by Lemma $1, C_{r t} * \bar{K}_{2}$ has a decomposition into sunlet graphs $L_{2 r t}=L_{q}$.

Subcase $2.2(m \equiv 0(\bmod 4))$. Set $m=2 t$. The graph $C_{r} * \bar{K}_{t}$ decomposes into Hamilton cycle $C_{r t}$ by [11]. Next, make each cycle $C_{r t}$ into $C_{r t} * \bar{K}_{2}$. Each graph $C_{r t} * \bar{K}_{2}$ decomposes into sunlet graph $L_{2 r t}$ by Lemma 1.
Theorem 3. Let $r, m$ be positive integers satisfying $r, m \equiv$ $0(\bmod 4)$, then $L_{r}$ decomposes $C_{r} * \bar{K}_{m}$.

Proof. Let the partite sets (layers) of the $r$-partite graph $C_{r} *$ $\bar{K}_{m}$ be $U_{1}, U_{2}, \ldots, U_{r}$. Set $m=2 t$. Obtain a new graph from $C_{r} * \bar{K}_{m}$ as follows.

Identify the subsets of vertices $\left\{x_{i, j}\right\}$, for $1 \leq i \leq r$ and $1 \leq j \leq m / 2$ into new vertices $x_{i}^{1}$, and identify the subset of vertices $\left\{x_{i, j}\right\}$ for $1 \leq i \leq r$ and $m / 2+1 \leq j \leq m$ into new vertices $x_{i}^{2}$ and two of these vertices $x_{i}^{k}$, where $k=1,2$, are adjacent if and only if the corresponding subsets of vertices in $C_{r} * \bar{K}_{m}$ induce $K_{t, t}$. The resulting graph is isomorphic to $C_{r} * \bar{K}_{2}$. Next, decompose $C_{r} * \bar{K}_{2}$ into cycles $C_{r / 2}$ as follows: $x_{k, 1} x_{k+1,1}, \ldots, x_{d, 1} x_{d-1,2}, \ldots, x_{k+1,2}, x_{k, 1}$

$$
k=1, \frac{r}{4}+1, \frac{r}{2}+1, \frac{3 r}{4}+1, \ldots, r-\frac{r}{4}+1, \quad d=\frac{r}{4}+k,
$$

where $k, d$ are calculated modulo $r$.

To construct the remaining cycles, apply mapping $\phi$ defined on the vertices.

Subcase 1.1 ( $i$ odd in each cycle). Consider

$$
\phi\left(x_{i, j}\right)=x_{i, j+1}
$$

This is the desired decomposition of $C_{r} * \bar{K}_{2}$ into cycles $C_{r / 2}$. Subcase 1.2 ( $i$ even in each cycle). Consider

$$
\phi\left(x_{i, j}\right)=x_{i, j}
$$

This is the desired decomposition of $C_{r} * \bar{K}_{2}$ into cycles $C_{r / 2}$.

By lifting back these cycles $C_{r / 2}$ of $C_{r} * \bar{K}_{2}$ to $C_{r} * \bar{K}_{2 t}$, we get edge-disjoint subgraphs isomorphic to $C_{r / 2} * \bar{K}_{t}$. Obtain a new graph again from $C_{r / 2} * \bar{K}_{t}$ as follows.

For each $j, 1 \leq j \leq t / 2$, identify the subsets of vertices $\left\{x_{i, 2 j-1}, x_{i, 2 j}\right\}$, where $1 \leq i \leq r / 2$ into new vertices $x_{i}^{j}$, and two of these vertices $x_{i}^{j}$ are adjacent if and only if the corresponding subsets of vertices in $C_{r / 2} * \bar{K}_{t}$ induce $K_{2,2}$. The resulting graph is isomorphic to $C_{r / 2} * \bar{K}_{t / 2}$. Then, decompose $C_{r / 2} * \bar{K}_{t / 2}$ into cycles $C_{r / 2}$. Each $C_{r / 2} * \bar{K}_{t / 2}$ decomposes into cycles $C_{r / 2}$ by [12]. By lifting back these cycles $C_{r / 2}$ of $C_{r / 2} *$ $\bar{K}_{t / 2}$ to $C_{r / 2} * \bar{K}_{t}$, we get edge-disjoint subgraph isomorphic to $C_{r / 2} * \bar{K}_{2}$. Finally, each $C_{r / 2} * \bar{K}_{2}$ decomposes into two sunlet graphs $L_{r}$ (by Lemma 1), and we have $C_{r} * \bar{K}_{m}$ decomposing into sunlet graphs $L_{r}$ as required.

Theorem 4 (see [12]). The cycle $C_{m}$ decomposes $C_{k} * \bar{K}_{m}$ for every even $m>3$.

Theorem 5 (see [12]). If $m$ and $k \geq 3$ are odd integers, then $C_{m}$ decomposes $C_{k} * \bar{K}_{m}$. 
Theorem 6. The sunlet graph $L_{m}$ decomposes $C_{r} * \bar{K}_{m}$ if and only if either one of the following conditions is satisfied.

(1) $r$ is a positive odd integer, and $m$ is a positive even integer.

(2) $r, m$ are positive even integers with $m \equiv 0(\bmod 4)$.

Proof. (1) Set $m=2 t$, where $t$ is a positive integer. Let the partite sets (layers) of the $r$-partite graph $C_{r} * \bar{K}_{m}$ be $U_{1}, U_{2}, \ldots, U_{r}$. For each $j$, where $1 \leq j \leq t$, identify the subsets of vertices $\left\{x_{i, 2 j-1}, x_{i, 2 j}\right\}$, for $1 \leq i \leq r$ into new vertices $x_{i}^{j}$, and two of these vertices $x_{i}^{j}$ are adjacent if and only if the corresponding subsets of vertices in $C_{r} * \bar{K}_{m}$ induce $K_{2,2}$. The resulting graph is isomorphic to $C_{r} * \bar{K}_{t}$. Then, decompose $C_{r} * \bar{K}_{t}$ into cycles $C_{t}$, where $t$ is a positive integer.

Now, $C_{t} \mid C_{r} * \bar{K}_{t}$ by Theorems 4 and 5 .

By lifting back these $t$-cycles of $C_{r} * \bar{K}_{t}$ to $C_{r} * \bar{K}_{2 t}$, we get edge-disjoint subgraphs isomorphic to $C_{t} * \bar{K}_{2}$. Each copy of $C_{t} * \bar{K}_{2}$ decomposes into sunlet graphs of length $2 t$ (by Lemma 1), and we have $C_{r} * \bar{K}_{m}$ decomposing into sunlet graphs of length $m$ as required.

(2) Set $m=2 t$, where $t$ is an even integer since $m \equiv$ $0(\bmod 4)$.

Obtain a new graph $C_{r} * \bar{K}_{t}$ from the graph $C_{r} * \bar{K}_{m}$ as in Case 1. By Theorem 4, $C_{t} \mid C_{r} * \bar{K}_{t}$. By lifting back these $t$-cycles of $C_{r} * \bar{K}_{t}$ to $C_{r} * \bar{K}_{2 t}$, we get edge-disjoint subgraphs isomorphic to $C_{t} * \bar{K}_{2}$. Each copy of $C_{t} * \bar{K}_{2}$ decomposes into sunlet graph of length $2 t$ (by Lemma 1). Therefore, $L_{m}$ | $C_{r} * \bar{K}_{m}$ as required.

Remark 7. In [10], it was shown that

$$
L_{2 r} * \bar{K}_{l} \text { can be decomposed into } l^{2} \text { copies of } L_{2 r} \text {. }
$$

This, coupled with Lemma 1, gives the following.

Theorem 8 (see [10]). The graph $C_{r} * \bar{K}_{2 l}$ decomposes into sunlet graphs $L_{2 r}$ for any positive integer $l$.

Lemma 9 (see [3]). Let $n \geq 4$ be an even integer. Then, $K_{n}+I$ is $C_{n}$-decomposable.

Lemma 10 (see [3]). Let $m$ and $n$ be integers with $m$ odd, $n \equiv$ $2(\bmod 4), 3 \leq m \leq n<2 m$, and $n^{2} \equiv 0(\bmod 2 m)$. Then, $K_{n}+I$ is $C_{m}$-decomposable.

Lemma 11 (see [3]). Let $m$ and $n$ be integers with $m$ odd, $n \equiv$ $0(\bmod 4), 3 \leq m \leq n<2 m$, and $n^{2} \equiv 0(\bmod 2 m)$. Then, $K_{n}+I$ is $C_{m}$-decomposable.

We can now prove the major result.

Theorem 12. For any even integers $m \geq 2, n>2$, and $q \geq$ 6 , the sunlet graph $L_{q}$ decomposes $K_{n}+I * \bar{K}_{m}$ if and only if $n^{2} m^{2} / 2 \equiv 0(\bmod q)$.
Proof. The necessity of the condition is obvious, and so we need only to prove its sufficiency. We split the problem into the following two cases.

Case $1(q \mid n)$

Subcase $1.1(n>q)$. Cycle $C_{n}$ decomposes $K_{n}+I$ by Lemma 9 , and we have

$$
C_{n} * \bar{K}_{m} \mid K_{n}+I * \bar{K}_{m} .
$$

Each graph $C_{n} * \bar{K}_{m}$ decomposes into sunlet graph $L_{q}$, where $q=n m$ by Lemma 2 , and we have $K_{n}+I * \bar{K}_{m}$ decomposing into sunlet graph $L_{q}$, where $q>n$.

Subcase $1.2(q=n)$. First, consider $n \equiv 0(\bmod 4)$.

Cycle $C_{q}$ decomposes $K_{q}+I$ by Lemma 9 , and we have

$$
C_{q} * \bar{K}_{m} \mid K_{q}+I * \bar{K}_{m} .
$$

Now, sunlet graph $L_{q} \mid\left(C_{q} * \bar{K}_{m}\right)$ by Theorem 3, and hence sunlet graph $L_{q}$ decomposes $K_{n}+I * \bar{K}_{m}$.

Also, consider $n \equiv 2(\bmod 4)$.

Suppose $m=2 t$. Cycle $C_{q / 2}$ decomposes $K_{q}+I$ by Lemma 10, and we have

$$
C_{q / 2} * \bar{K}_{2 t} \mid K_{q}+I * \bar{K}_{2 t} .
$$

Now, sunlet graph $L_{q}$ decomposes $C_{q / 2} * \bar{K}_{2 t}$ by Theorem 8 , and we have $K_{n}+I * \bar{K}_{m}$ decomposing into sunlet graph of length $q$.

Case $2(q \mid m)$

Subcase $2.1(m \equiv 0(\bmod 4))$. Suppose $m=q$, and by Lemma 9 , cycle $C_{n}$ decomposes $K_{n}+I$, and we have

$$
C_{n} * \bar{K}_{q} \mid K_{n}+I * \bar{K}_{q} .
$$

Also, sunlet graph $L_{q}$ decomposes each $C_{n} * \bar{K}_{q}$ by Theorem 6, and we have sunlet graph $L_{q}$ decomposing $K_{n}+I * \bar{K}_{m}$.

Subcase $2.2(m \equiv 2(\bmod 4))$. Let $m=q$ and $r \leq n$ an odd integer. Cycle $C_{r}$ decomposes $K_{n}+I$, by Lemmas 9,10 , and 11, and we have

$$
C_{r} * \bar{K}_{q} \mid K_{n}+I * \bar{K}_{q} .
$$

Now, each $C_{r} * \bar{K}_{q}$ decomposes into sunlet graph $L_{q}$ by Theorem 6, and we have $K_{n}+I * \bar{K}_{m}$ decomposing into sunlet graph $L_{q}$ as required.

Subcase $2.3(m>q)$. Set $m=w q$, where $w$ is any positive integer, then by Subcases 2.1 and 2.2, we have

$$
L_{q} * \bar{K}_{w} \mid\left(K_{n}+I * \bar{K}_{q}\right) * \bar{K}_{w} .
$$

Each graph $L_{q} * \bar{K}_{w}$ decomposes into sunlet graph $L_{q}$ by Remark 7, and we have $K_{n}+I * \bar{K}_{m}$ decomposing into sunlet graph $L_{q}$. 


\section{References}

[1] B. Alspach and H. Gavlas, "Cycle Decompositions of $K_{n}$ and $K_{n}$-I," Journal of Combinatorial Theory B, vol. 81, no. 1, pp. 77-99, 2001.

[2] M. Šajna, "Cycle decompositions. III. Complete graphs and fixed length cycles," Journal of Combinatorial Designs, vol. 10, no. 1, pp. 27-78, 2002.

[3] M. Šajna, "Decomposition of the complete graph plus a 1-factor into cycles of equal length," Journal of Combinatorial Designs, vol. 11, no. 3, pp. 170-207, 2003.

[4] D. G. Hoffman, C. C. Lindner, and C. A. Rodger, "On the construction of odd cycle systems," Journal of Graph Theory, vol. 13, no. 4, pp. 417-426, 1989.

[5] R. S. Manikandan and P. Paulraja, " $C_{p}$-decompositions of some regular graphs,” Discrete Mathematics, vol. 306, no. 4, pp. 429451, 2006.

[6] R. S. Manikandan and P. Paulraja, " $C_{5}$-decompositions of the tensor product of complete graphs," The Australasian Journal of Combinatorics, vol. 37, pp. 285-293, 2007.

[7] B. R. Smith, "Decomposing complete equipartite graphs into cycles of length 2p," Journal of Combinatorial Designs, vol. 16, no. 3, pp. 244-252, 2008.

[8] B. R. Smith, "Complete equipartite 3p-cycle systems," The Australasian Journal of Combinatorics, vol. 45, pp. 125-138, 2009.

[9] R. Anitha and R. S. Lekshmi, "N-sun decomposition of complete, complete bipartite and some Harary graphs," International Journal of Mathematics Sciences, vol. 2, no. 1, pp. 33-38, 2008.

[10] A. D. Akwu and D. O. A. Ajayi, "Decomposing equipartite graphs into certain corona graphs of length $2 p$ ".

[11] R. Laskar, "Decomposition of some composite graphs into Hamilton Cycles," in Proceedings of the 5th Hungarian Colloquium keszthely, pp. 705-716, North Holland, 1978.

[12] D. Fronček, P. Kovář, and M. Kubesa, "Decompositions of complete graphs into blown-up cycles $C_{m}$ [2]," Discrete Mathematics, vol. 310, no. 5, pp. 1003-1015, 2010. 


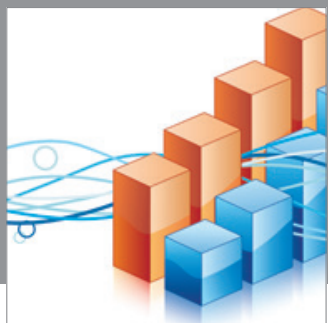

Advances in

Operations Research

mansans

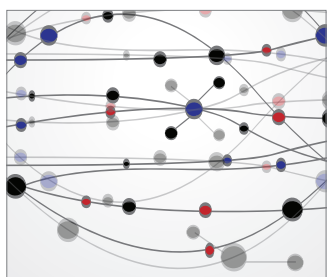

The Scientific World Journal
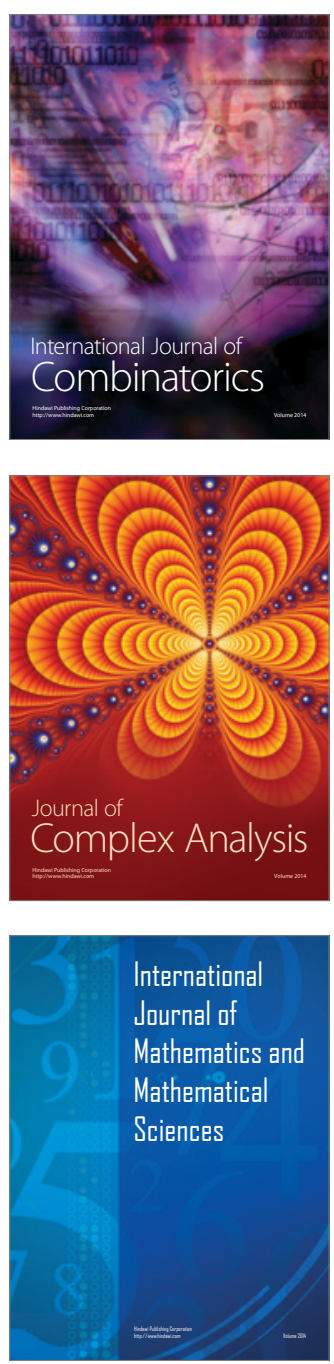
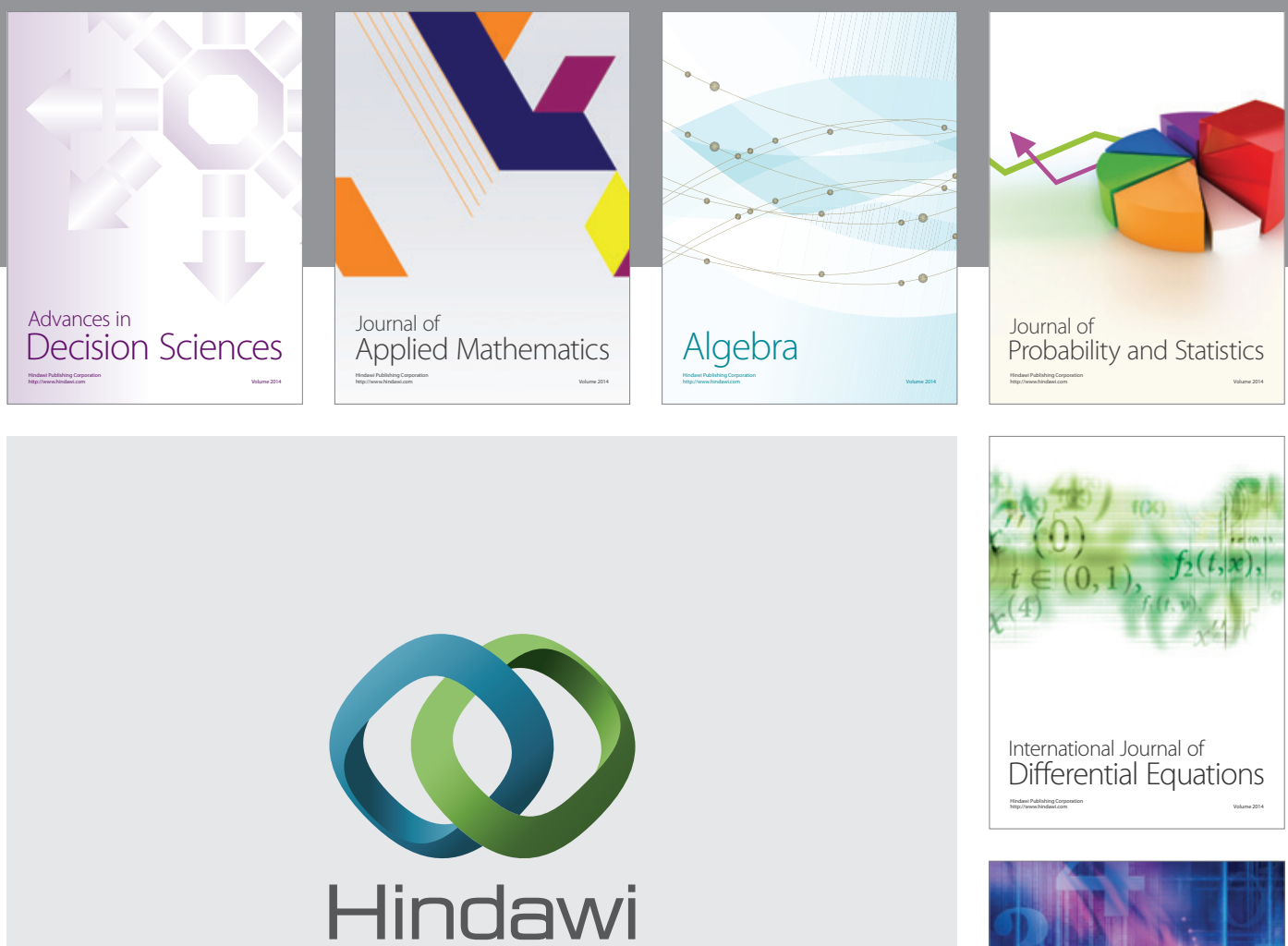

Submit your manuscripts at http://www.hindawi.com
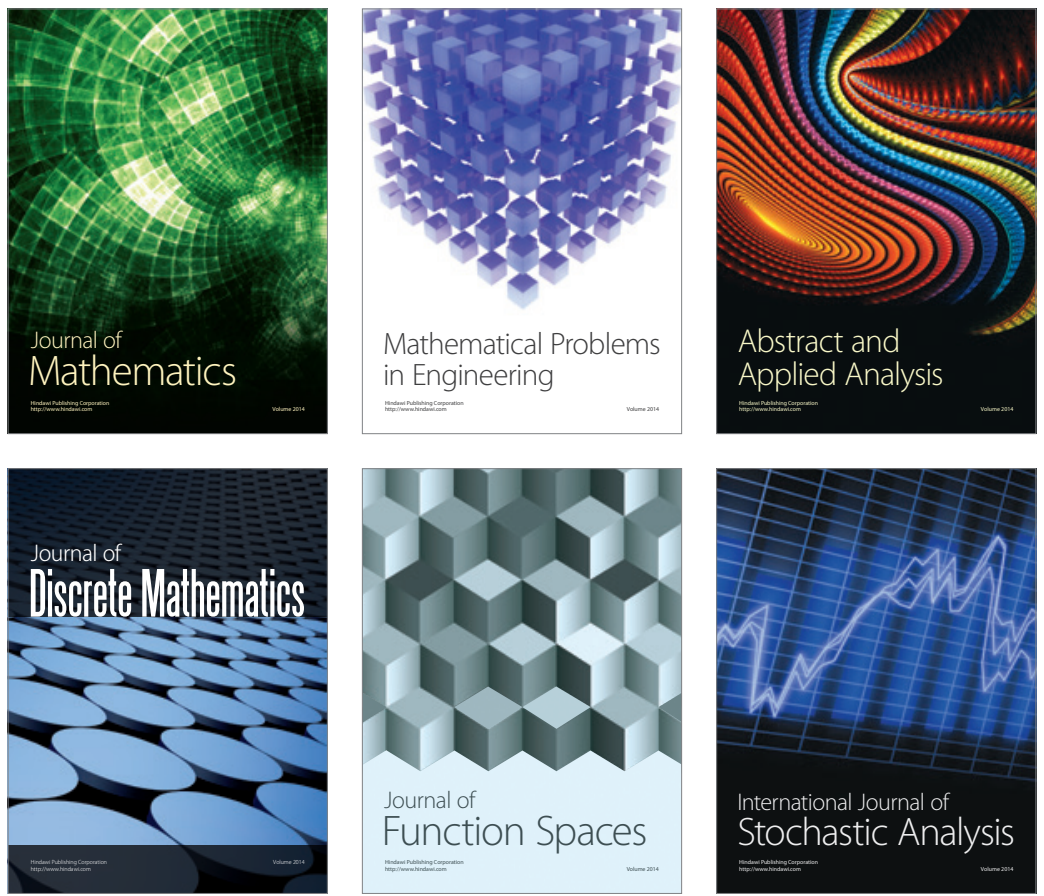

Journal of

Function Spaces

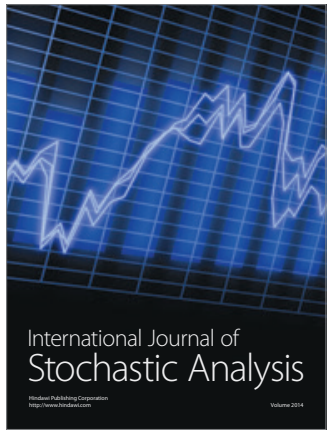

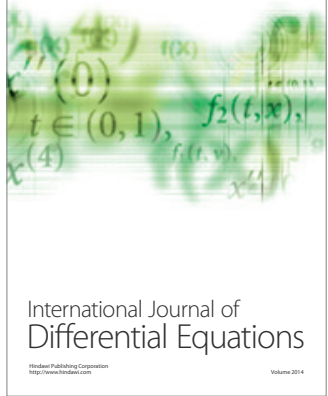
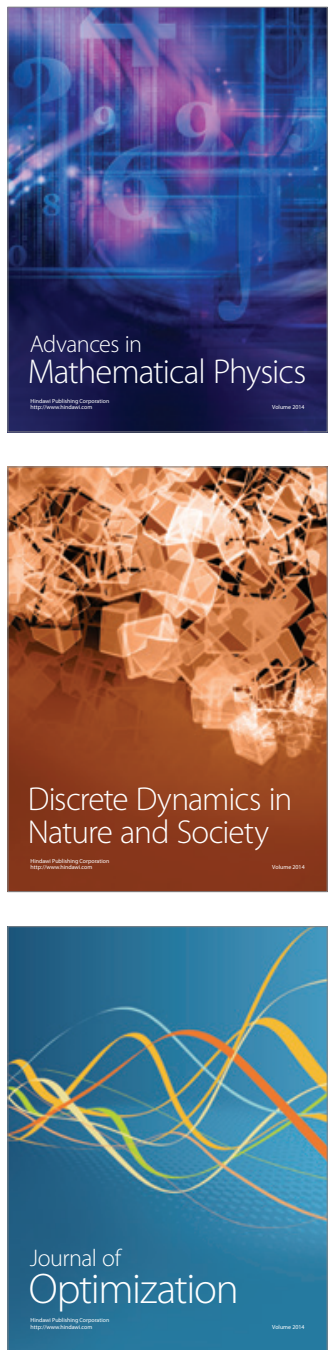